\title{
Contextual effects in latent inhibition with an appetitive conditioning procedure
}

\author{
STEPHEN CHANNELL and GEOFFREY HALL \\ University of York, York, England
}

\begin{abstract}
In three experiments, rats received presentations of a diffuse 30-sec stimulus (a light) and of food, and their tendency to enter the food tray was monitored. Experiment 1 showed that when the light was made to signal the delivery of food, the response of entering the food tray increased in frequency during the stimulus. The acquisition of this conditioned response to the light was retarded in subjects that had received preexposure to the stimulus. In Experiments 2 and 3, subjects received preexposure to the stimulus, some in the same context as that subsequently used for stimulus-food pairings and some in a different context. Those experiencing the change of context acquired the response more readily. It is argued that these results demonstrate a latent inhibition effect that is attenuated by contextual change.
\end{abstract}

Recent theories of latent inhibition have supposed that prior exposure to a to-be-conditioned stimulus retards subsequent conditioning by reducing the associability or conditionability of the stimulus. They also propose that the mechanism responsible obeys laws similar to those that govern conditioning itself (Lubow, Schnur, \& Rifkin, 1976; Pearce \& Hall, 1980; Wagner, 1976). For many experimental demonstrations of latent inhibition, however, an alternative account is available. When the target stimulus is first presented during the preexposure phase of such an experiment, it will evoke some unconditioned response (UR) that will habituate with repeated presentations of the stimulus. The UR will occur, however, in control subjects when they experience the stimulus for the first time at the start of conditioning. When the UR and the conditioned response (CR) are similar in form, this may in itself be enough to produce the appearance of rapid acquisition of the CR by the control subjects.

This problem is particularly acute for those experimental studies that have tried to investigate the role of the context in which training takes place. It has been suggested (Wagner, 1976, 1978, 1979) that the loss of associability suffered by a to-be-conditioned stimulus (CS) during preexposure may, at least to some extent, be specific to the context in which the preexposure is given. Animals given preexposure in one context and then conditioned with the same CS in a different context should, according to this view, acquire the CR more readily than subjects that experience both phases of training in the same context.

This work was supported by a grant from the United Kingdom Science and Engineering Research Council. We thank T. Dixon for his assistance. Reprint requests may be sent to $G$. Hall, Department of Psychology, University of York, York YO1 SDD, England.
Most of the available experiments that allow an investigation of this proposition (e.g., Anderson, O'Farrell, Formica, \& Caponigri, 1969; Anderson, Wolf, \& Sullivan, 1969; Dexter \& Merrill, 1969; Hall \& Minor, 1983) have used aversive conditioning procedures in which the associative strength acquired by the CS was assessed by its ability to suppress appetitively maintained responding. But the stimuli used in these experiments will commonly produce a suppression of responding, even when they lack associative strength. The response (perhaps freezing) that is responsible for this unconditioned suppression will habituate with repeated presentations of the stimulus, but there is plentiful evidence (e.g., Wagner, 1976) that some small change in the nature of the stimulus will restore a habituated response. Presenting a familiar stimulus in a new context may allow dishabituation, and thus the suppression evoked by such a stimulus might include a proportion contributed by the reinstated UR. It would not, therefore, be an accurate measure of the associative strength governed by the stimulus. Studies of latent inhibition need to control for these possibilities.

This is not to say that the latent inhibition phenomenon is quite distinct from that of habituation. The preexposure phase of a latent inhibition procedure is identical in procedure to that of a habituation experiment, and the retarded conditioning that results has often been viewed as being the result of the habituation of some observing or attentional response. But there may be other responses whose habituation (or dishabituation) could be confounded with differences in the probability of the $C R$, making the latter an unreliable index of the associability of the stimulus in question (cf. Sharp, James, \& Wagner, 1980).

Several experimental strategies have been tried in an attempt to unconfound the various possible ef- 
fects of preexposure (see, e.g., Carlton \& Vogel, 1967; Domjan \& Siegel, 1971; Lubow, Markman, \& Allen, 1968). Perhaps the most satisfactory strategy is that used by Halgren (1974). In Halgren's experiment, rats were required to learn a successive discrimination, half the subjects learning to press a lever in the presence of a tone for sucrose reward, and half learning to press in the absence of the tone. For all subjects, it was found that the formation of the discrimination was retarded by prior exposure to the tone. Whatever the initial UR to the tone, it is difficult to argue that this response could both promote leverpressing when the tone is a positive discriminative stimulus and also interfere with it when the tone is a negative stimulus. This experiment (see also the related work of Reiss \& Wagner, 1972, and Rescorla, 1971) allows the conclusion that a change in the associability of the stimulus is among the effects produced by the habituation procedure. It remains to use an equivalent experimental technique in order to demonstrate that the effects produced by change of context are also effects on associability.

The experiments reported below made use of a CS that tends to suppress appetitively maintained responding when first presented. They also used an appetitive conditioning procedure in which the $\mathbf{C R}$ measured was an increase in responding in the presence of the CS. Relatively rapid learning about a novel stimulus (or about a familiar stimulus presented in a new context) could not be due, therefore, to the summation of the CR and the UR. Since Halgren's is one of the few experiments on latent inhibition to employ an appetitive reinforcer (see also Lubow, Rifkin, \& Alek, 1976, and Tranberg \& Rilling, 1978), the aim of Experiment 1 was to provide a demonstration of the effect with rats trained by an appetitive conditioning procedure more closely similar to the simple conditioning techniques used in previous work with aversive reinforcers (Lubow, 1973). Experiments 2 and 3 went on to investigate the effects of changing the context between preexposure and conditioning phases.

\section{EXPERIMENT 1}

The aim of this experiment was to provide an unambiguous demonstration of latent inhibition using an appetitive conditioning procedure. There were two groups of subjects. One received preliminary exposure to a number of presentations of a stimulus (the illumination of the experimental chamber), and the second group did not. Both groups then received training in which the presentation of the light was followed by the delivery of food. The response measured was the tendency of the subjects to approach the site of food delivery, during the CS and before the delivery of food.
Our training procedure was based on the results of a preliminary experiment and was adapted from that used by Holland (e.g., 1977, 1979). Holland has shown that changes in rats' behavior occur during a stimulus (such as a light or tone) that signals the delivery of food. In the preliminary experiment, we modified Holland's procedure as follows. The stimulus was $30 \mathrm{sec}$ in duration, rather than the $10 \mathrm{sec}$ used by Holland, allowing an increased time on each trial over which responses could be observed. Holland $(1977,1979)$ scored a wide range of responses, but we recorded only the behavior of entering the food tray. Our apparatus included a food tray protected by a hinged flap, the opening of which operated a microswitch. This provided a convenient and objective way of recording behavior. Holland (1977) found that the tendency to approach the food tray is just one of the patterns of behavior that change during conditioning, and that the extent to which this pattern comes to dominate depends upon the nature of the CS. Our stimulus (the general illumination of the chamber) was not well localized and might be expected to evoke the behavior of approaching the food tray (i.e., to produce goaltracking rather than sign-tracking; Boakes, 1977). This expectation was confirmed by the results of the pilot experiment. Four rats, given a total of 80 lightfood pairings over eight sessions, developed a high rate of flap-entry responding in the presence of the stimulus. Four control subjects, given uncorrelated presentations of the light and of food, showed response rates that were no higher in the presence of the stimulus than in its absence.

Our preliminary experiment also showed that the unconditioned effect of the light was to bring about a suppression of flap-entry responding. For the first session in which the light was presented, the mean baseline rate (i.e., the rate of response in the absence of the light) was 3.20 responses/min; the rate in the presence of the light was 1.63 responses/min. Thus, the UR to the light is opposed to the CR that develops with light-food pairings. A superiority of control subjects over preexposed subjects in the present experiment could not, therefore, be ascribed to the summation of the UR and the CR. Any tendency for the UR to habituate during preexposure should help rather than hinder the emergence of the flap-entry response during conditioning for the preexposed group.

\footnotetext{
Method

Subjects. The subjects were 20 naive female hooded rats. They were maintained at $85 \%$ of their free-feedings weights by daily weighing and controlled feeding.

Apparatus. Two identical Skinner boxes were used, each $20 \times$ $24 \times 23 \mathrm{~cm}$ and housed in a sound-attenuating chamber. The retractable levers were withdrawn throughout the experiment. Food reinforcement consisted of a single $45-\mathrm{mg}$ pellet delivered to a
} 
recessed food tray. The entrance to the tray was at floor level and centrally positioned on one wall of the box. It was covered by a flap of transparent plastic, $6 \mathrm{~cm}$ high $\times 5 \mathrm{~cm}$ wide, that had to be pushed for the food pellet to be retrieved. Openings of the flap operated a microswitch. The box was equipped with an overhead 30-W strip-light above the white translucent plastic ceiling; this light was operated at $100 \mathrm{~V}$ to supply the light CS. An exhaust fan in the chamber provided a background noise of $65 \mathrm{~dB}$.

Procedure. The first two sessions consisted of magazine training with food pellets delivered according to a variable time 60 -sec schedule. Both sessions were $\mathbf{3 0}$ min long. During the first of these sessions, the flap in front of the food tray was taped back, but in the second session it was necessary to push back the flap to retrieve the food. All subjects learned to do this.

All subjects then received six daily 44-min sessions, during which they were placed in the apparatus but no food was delivered. Half of the animals were allocated to the preexposure condition. For these subjects, the 30-sec light was presented 10 times in each session according to a variable interval 4-min schedule. Subjects in the control condition experienced no programmed stimuli during this stage. Ten conditioning sessions followed, in which trials for the preexposed group continued just as before, except that a single food pellet was delivered coincidentally with the offset of the light. Control subjects received identical treatment. They experienced the light for the first time at this stage.

Flap-entries were recorded separately during the presence and the absence of the stimulus. To attenuate the effects produced by individual differences in the rate of responses, these results were expressed as a ratio score having the form $A /(A+B)$, where $A$ represents the number of responses occurring during the $C S$ and $B$ the number occurring in a $30-\mathrm{sec}$ period immediately preceding the onset of the stimulus. Ratio scores above .50 indicate an increase in the rate of flap-entry during the stimulus. Response rates on all trials each day were pooled before the ratio was calculated.

\section{Results and Discussion}

One subject in the control group failed to eat the food pellets during the conditioning sessions. The results for this subject were excluded from all analyses, as were those for the corresponding subject in the preexposed group. The mean scores presented below are thus based on groups of nine.

During the first session of the exposure phase, subjects in the preexposed group had a mean of 1.82 flap-entry responses/min, and those in the control group had a mean of 1.61 responses/min. The equivalent scores for the sixth and final session of this stage were .72 and .42 responses/min, a decline to be expected since no food was delivered during this stage. An analysis of variance carried out on the scores for all six sessions of this phase showed a significant change over sessions $[F(5,80)=9.20, p<$ $.01]$, but no effect of groups $[F(1,16)=1.29]$ and no significant interaction $(\mathrm{F}<1)$.

When it was first presented to the preexposed group, the light produced a suppression of responding, with all but one of the subjects having a ratio score below .50 (mean $=.16$ ) on the first session. By the final session of preexposure, the mean ratio had risen to .34, with four of the nine subjects having scores above .50 .

The results of the conditioning phase are shown in Figure 1. It is apparent that the introduction of the
CS-US contingency produced an increase in responding during the CS for both groups, and that this increase occurred more readily in subjects not familiar with the CS. An analysis of variance carried out on the data presented in the figure showed there to be a significant difference between the groups $[F(1,16)=5.39, p<.05]$, a significant change over sessions $[F(9,144)=15.79, p<.01]$, but the interaction between these factors fell short of significance $[F(9,144)=1.35]$. The mean rate of response recorded during prestimulus periods was 2.44 responses/min for the preexposed group and $1.41 \mathrm{re-}$ sponses/min for the control group. These rates did not differ significantly $[F(1,16)=3.75]$.

The acquisition curves of Figure 1 show features that have also been found in previous studies of latent inhibition: The superiority of the control group is evident quite early in training and is maintained until the scores of the two groups converge on a common asymptote (cf. Siegel, 1972). But, with this procedure, the anticipated difference between the groups is not present on the first block of trials as commonly happens with procedures in which conditioning is indexed by the acquisition of suppression (e.g., Hall \& Pearce, 1979; Rudy, Krauter, \& Gaffuri, 1976). The difference between the groups cannot, therefore, be ascribed to the habituation of the UR in the preexposed group, since such a change in response pattern would help rather than hinder the performance of the CR in this experiment.

It should be added that, although the conditioning procedure used in this experiment was basically Pavlovian (the response has been referred to as a $\mathrm{CR})$, it cannot be assumed that an association was formed between stimulus and food. Flap-pushing can be interpreted as being an instrumental response (reinforced by access to food) that comes under the stimulus control of the light. Indeed, Holland (1979)

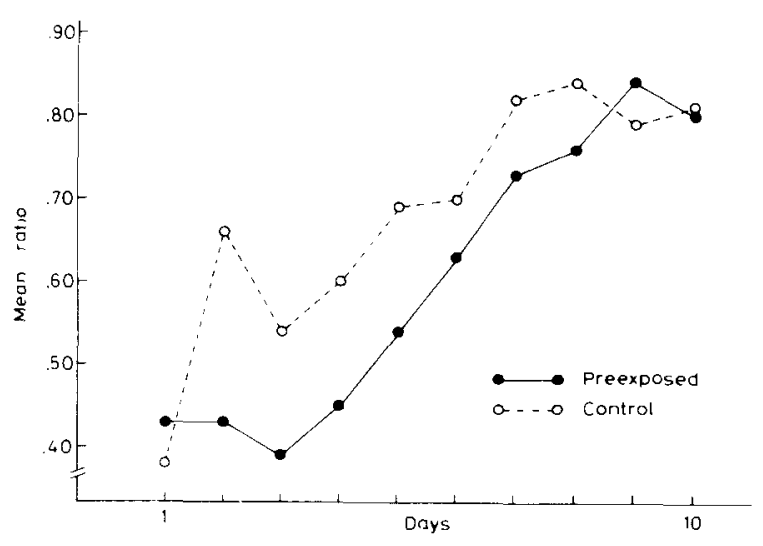

Figure 1. Experiment 1. Conditioning phase: Flap-entry responses during the light expressed as a ratio of baseline reaponse rate. Preexposed subjects had received 60 prior exposures to the Ilght. 
has demonstrated that imposing an omission contingency for magazine behavior (i.e., omitting the delivery of food when the rat stood with its nose in the food tray) tended to eliminate this behavior. Such evidence is not conclusive (Mackintosh \& Dickinson, 1979), but it is consistent with the possibility that the response-reinforcer contingency plays some part in maintaining the behavior. The issue is difficult to resolve, but uncertainty about it is no real problem for our present purposes. It is still possible to investigate latent inhibition using this conditioning paradigm even though we do not know whether the stimulus in question is functioning as a classical CS or as a discriminative stimulus.

\section{EXPERIMENT 2}

In this experiment, two groups of subjects were given preexposure to the to-be-conditioned stimulus, each group in its own distinctive environment. All animals then received conditioning trials with this stimulus, half of the subjects receiving training in the context with which they were familiar and half in the other context. If latent inhibition is context specific, those experiencing the change of context should learn more readily.

It was necessary, therefore, to provide two contexts that were discriminably different to the rat. Dexter and Merrill (1969) have shown that rats can discriminate between chambers differing in level of background illumination, color, level of masking noise, flooring material, and position of lever. Not all these dimensions were available to us (e.g., there was no lever present in our test chambers). Furthermore, since our CS was visual (Dexter \& Merrill used an auditory $C S$ ), we did not want to vary the background level of illumination. Accordingly, we used test chambers that differed in the level of backsround noise and also in the odor, produced by treating the floors of the chambers with highly scented fluids. The ease with which rats can be conditioned to tastes and odors perhaps suggests that this latter feature might be salient for these animals. The specific odors used were chosen because they were readily available commercially and were distinctive, at least to the human nose.

\section{Method}

The subjects were 16 naive male hooded rats maintained at $80 \%$ of their free-feeding weights. (Channell \& Hall, 1981, have found this deprivation level to be roughly functionslly equivalent to the $85 \%$ level used for the female subjects in Experiment 1.) The apparatus was that used in the preceding experiment. In one Skinner box (box A), white noise at an intensity of $70 \mathrm{~dB}$ was presented continuously. In both boxes, the tray beneath the grid floor of the chamber was lined with absorbent paper on which approximately $2 \mathrm{ml}$ of scented fluid was sprinkled. For box $A$, the odor was of "mint" (peppermint essence); for box B, it was of "violets" (produced by a scented proprietary cleaning fluid). The lining paper was replaced and the fluids renewed a few minutes before the start of each training session.

Half the animals were assigned at the outset to box $A$, and half to box B. After magazine training, all animals received an exposure phase of training lasting six sessions. No food was delivered, and all subjects received 10 presentations of the 30 -sec light in each session. There followed 10 sessions of appetitive conditioning organized in the same general way as in the preceding experiment. Half of the subjects preexposed in box $A$ were conditioned in box $B$; half of those preexposed in $B$ were conditioned in A. The remainder experienced no change of context. Thus there were two main experimental groups: those preexposed and conditioned in the same context and those experiencing a change of context between the phases of training.

Procedural details not given above were the same as for Experiment $\mathrm{J}$.

\section{Results and Discussion}

The mean rate of flap-entry responding over all 6 days of the preexposure phase was .46 responses $/ \mathrm{min}$ for the subjects in box $A$ and .60 responses $/ \mathrm{min}$ for the subjects in box $B$. These rates did not differ significantly $[F(14)=1.13]$. The light tended to suppress responding when first presented. The mean ratio score for all 16 animals over the first 10-trial preexposure session was .18. Only two animals showed an elevation, one trained in box $A$ and one in box $B$.

The results for the conditioning sessions are shown in Figure 2. There was no obvious difference between box $A$ and box $B$ in the rate at which conditioning occurred, and the results are pooled in the figure to produce means for two main groups, those conditioned in a familiar context and those conditioned in a new one. Both groups showed some initial suppression of responding. There was an increase in the rate of the flap-entry response during the CS as conditioning proceeded, and this increase occurred more readily in the subjects transferred for conditioning to a new context. An analysis of variance carried out on the data shown in the figure revealed a significant

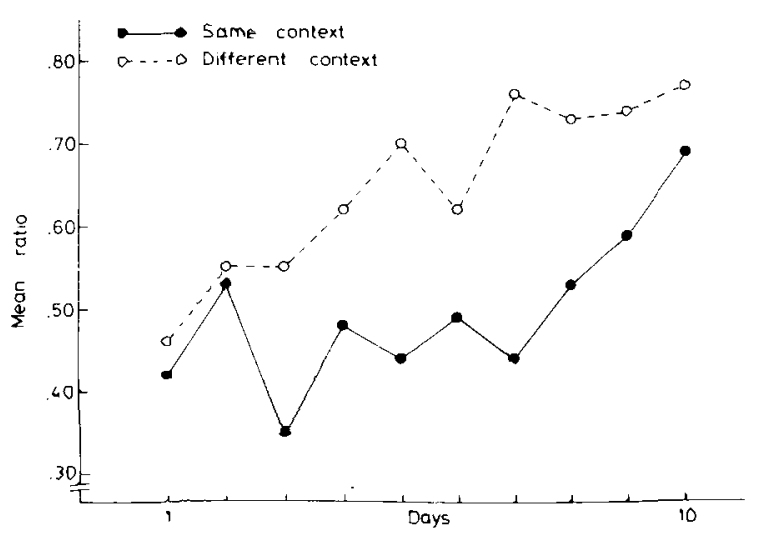

Figure 2. Flap-entry ratio scores for the conditioning phase of Experiment 2. All subjects had been preexposed to the Hight either in the same context as that used for conditioning or in different context. 
change over days $[F(9,144)=2.74, \mathrm{p}<.01]$ and a significant difference between the groups $[F(1,14)$ $=5.85, \mathrm{p}<.05]$. The interaction between these two factors was not significant $(F<1)$. The two groups did not differ in their baseline response rates during conditioning. The mean overall rate for the same-context group (calculated from responses emitted in prestimulus periods) was 1.09 responses/ min; that for the different-context group was 1.18 responses/min $(F<1)$.

Thus, animals preexposed to a stimulus in one context that subsequently are conditioned in a different context learn more readily than those receiving preexposure and conditioning in the same context. This finding demonstrates that our procedures, which were intended to produce two contexts discriminable by rats, were in fact successful. It also supplies preliminary evidence consistent with the view that the latent inhibition effect is context specific. But before accepting this conclusion, further control procedures are needed.

\section{EXPERIMENT 3}

The results of Experiment 2 suggest that the retardation of subsequent conditioning normally produced by preexposure to the stimulus can be attenuated or abolished by a change in context. But it is also possible that the results have nothing to do with latent inhibition; perhaps a change of context from $A$ to $B$, or vice versa, is itself enough to promote conditioning. Accordingly, four groups of subjects were included in this experiment. Two were treated in the same way as the subjects in Experiment 2; they constituted the preexposed same-context and preexposed different-context groups. The other two groups, control same-context and differentcontext, were treated identically except that they received no preexposure to the stimulus. Any advantage bestowed simply by changing the context would show up as superior learning in the control different-context group in comparison with the other control group.

\section{Method}

The subjects were 32 naive male hooded rats maintained as in the previous experiments. The apparatus was the same as that used in Experiment 2 except that four rather than two Skinner boxes were available. Two of these were treated in the same way as box $\mathrm{A}$ of Experiment 2, and two were treated as box B had been.

After preliminary magazine training, the subjects were assigned at random to form four equal-sized groups. All received six preexposure sessions, half the animals in context $A$ and half in context $B$. For the 16 subjects in the preexposure condition, the 30 sec light was presented 10 times during each session. No food was delivered. Control subjects did not experience the light.

There followed 12 sessions of conditioning in which, for all subjects, the light preceded the delivery of a food pellet. Half of the preexposed and half of the control subjects received conditioning in the same context as before; the remainder experienced a change of context. For the subjects experiencing a change of context, half in each major group shifted from $B$ to $A$ and half from $A$ to $B$. Conditioning was assessed, as before, by means of a ratio score, but in this experiment the baseline rate was derived from the total number of responses emitted in the absence of the CS throughout the session. In all other respects, the procedures used in this experiment were the same as those described for Experiment 2.

\section{Results and Discussion}

The mean baseline rates of flap-entry responding during the preexposure phase were as follows: 1.21 responses/min for rats preexposed to the stimulus in context $\mathrm{A}, 0.75$ responses $/ \mathrm{min}$ for those preexposed in context $\mathrm{B}, 1.59$ responses/min for the control subjects placed in context $A$, and 1.36 responses $/ \mathrm{min}$ for the control subjects in context B. An analysis of variance revealed no significant difference among these scores $[F(3,28)=2.43]$. When first presented to the preexposed groups, the light produced suppression of responding. The mean suppression ratio for the first session of the exposure phase was .23 for the subjects in context $\mathrm{A}$ and .28 for the subjects in context B. One subject in each group had a ratio score above .50 .

The results for the conditioning phase were reduced to two-session blocks, the scores from both days being pooled before individual ratios were calculated. Figure 3 shows the acquisition of the CR by the four major groups. It shows that, for all groups, the ratio score increased with training but that it did so at different rates in the various groups. An analysis of variance carried out on the data shown in the figure revealed no significant overall effect of groups $(F<1)$, but a significant effect of blocks $[F(5,140)=60.65, p<.01]$ and a significant interaction between these factors $[F(15,140)=1.95, p<$ $.05]$. This interaction was not produced by differences in the baseline rate of responding. Certainly, the baseline rate tended to decline over the course

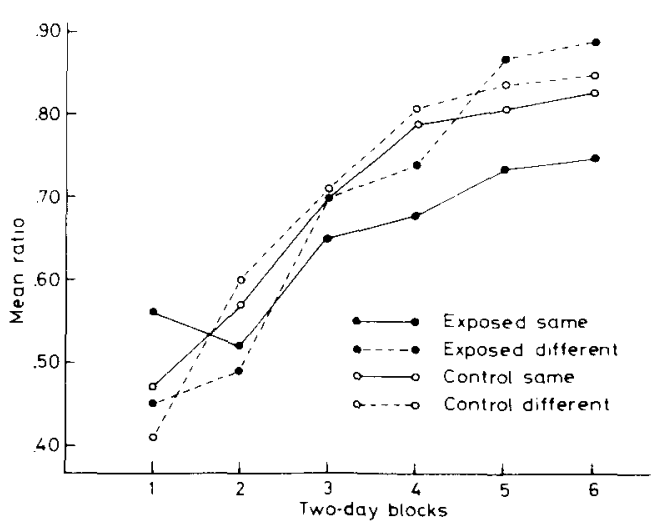

Figure 3. Flap-entry scores for the conditioning phnse of Experiment 3. Hall the subjects experienced a change of context for conditioning and half experienced a familiar context. Half of each of these groups had also recelved prior exposure to the stimulus. 
of conditioning (the mean overall rate was 3.03 responses/min on the first block and 1.98 on the final block), but the decline was evident in all groups. An analysis of variance paralleling that done on the ratio scores, carried out using the baseline response rates, revealed a significant effect of blocks $[F(5,140)=$ $8.63, p<.01]$ but no significant difference among the four groups and no significant interaction ( $F<1$ in both cases).

Figure 3 suggests that the significant interaction in the ratio scores derived from relatively slow learning (compared with the other groups) by the group that received preexposure and conditioning in the same context. A further analysis of variance comparing this group with the subjects that received preexposure and a change of context showed no significant overall effect of blocks $[F(5,70)=24.13, p<$ $.01]$ and a significant interaction between groups and blocks $[F(5,70)=2.83, p<.05]$. This finding thus replicates that of Experiment 2. The performance of the control subjects that received no prior exposure to the stimulus demonstrates that the change of context in itself was not sufficient to produce an enhanced rate of learning. These two groups differed little during conditioning, and an analysis of variance comparing their performance over the six blocks showed only an effect of blocks $[F(5,70)=40.81$, $p<.01$, the difference between the groups and the interaction both being nonsignificant $(F<1$ in both cases). We conclude, therefore, that the change of context has its effect by eliminating the latent inhibition produced by preexposure to the stimulus.

\section{GENERAL DISCUSSION}

Experiment 1 showed that a light CS that initially evokes suppression of a flap-entry response, will come to govern an increased rate of this response when it signals the delivery of food. It also provided a demonstration of latent inhibition for this conditioning procedure. Experiments 2 and 3 showed that latent inhibition can be abolished by giving preexposure and conditioning in different contexts. This last effect was not produced by a reappearance of the habituated UR to the light consequent upon the change in context.

That latent inhibition might be context-specific is suggested by a number of previous studies, although the detailed procedures used here were rather different from those used previously. Thus, Willner (Note 1) has shown, for flavor aversion learning, that the latent inhibition produced by prior exposure to the flavor is attenuated, although not abolished, when the exposure is given in a quite different cage from that used in conditioning. Using an electric shock as the reinforcer, Anderson, O'Farrell, Formica, and Caponigri (1969) found that preex- posure to a buzzer in the same chamber as was subsequently used for conditioning produced more latent inhibition than did preexposure to the buzzer in some different cage. Both groups of subjects showed poorer conditioning, however, than subjects that had not previously experienced the CS. Finally, Hall and Minor (1983, Experiment 2), using a tone as the CS and shock as the US, found no evidence at all of latent inhibition in animals given preexposure to the tone in a different context. These subjects conditioned just as readily as subjects for whom the tone was novel. Both groups learned more readily than subjects preexposed and conditioned in the same context.

This pattern of results is anticipated by Wagner's (1979) general theory of conditioning. Wagner suggests that latent inhibition reflects the formation, during preexposure, of an association between the preexposed stimulus and the context in which it is presented. The existence of this association permits the presentation of the context to "prime" a representation of the stimulus into the short-term memory mechanism held to be responsible for associative learning. An event that has been primed in this way is thought to be less capable of forming an association with other events. It will be poor, therefore, at forming an association with the reinforcer when conditioning is given in the same context as was used for preexposure. But, if the context is changed at the start of the conditioning phase, priming will be less likely to occur and learning should proceed readily.

Wagner's account fits the results very well, but it is not the only interpretation available. Hall and Minor (1983) discuss a number of possible alternatives, and, therefore, we will restrict discussion to just one of them here. It assumes that contextual cues can come to form a part of the stimulus complex (i.e., to-be-conditioned stimulus plus context) to which latent inhibition accrues. A change in the context would then be functionally equivalent to a change in some feature of the CS itself, and latent inhibition is known to be specific to the stimulus used. Thus, Siegel (1969) has shown, for rabbits preexposed to a tone of a given frequency, that subsequent conditioning was most retarded when the same tone was used as the CS but was less retarded when a tone differing in frequency was used. Mackintosh (1973) has demonstrated, for rats, that the latent inhibition effect produced by preexposure to a light was much reduced when the light was presented in compound with a noise during preexposure. Wagner's theory is quite able to accommodate these findings (see Rudy et al., 1976), and there are currently no grounds on which to decide between the alternative interpretations. When such evidence is available, it may help us to choose between Wagner's (1979) 
account of latent inhibition, with its emphasis on the association between context and stimulus, and theories like those proposed by Mackintosh (1975) and by Pearce and Hall (1980) that give the context no such role.

Finally, it should be said that, although the experiments just described provided evidence for an attenuation or abolition of latent inhibition when the context was changed, they did not show a reversal of latent inhibition (i.e., a facilitation of acquisition after preexposure in a different context). Such a reversal was reported by Lubow, Rifkin, and Alek (1976), who used an odor as the CS and varied the shape of the cage in which it was presented in order to manipulate the context. They found learning to be most rapid when the rats experienced a familiar odor in a new context, these subjects learning even more readily than rats for whom the odor was quite novel. Again, Channell and Hall (1981) found that rats exposed to a pair of stimuli in their home cage showed a facilitation when subsequently required to learn a simultaneous visual discrimination between these stimuli in a jumping stand. The source of this facilitation is uncertain and presumably derives from some difference in procedure between the experiments just cited and the orthodox latent inhibition procedure used in the present experiments. The new results reported here do not narrow the range of possibilities much, but they do allow us to conclude that the use of an appetitive reinforcer (as was used in the experiments by Channell \& Hall, 1981, and Lubow, Rifkin, \& Alek, 1976) is not in itself enough to produce a reversal of latent inhibition when combined with a change in context.

\section{REFERENCE NOTE}

1. Willner, J. A. Spatial factors in latent inhibition. Paper presented at the meeting of the Eastern Psychological Association, Hartford, Connecticut, 1980.

\section{REFERENCES}

Anderson, D. C., O'F arrell, T., Formica, R., \& Caponiari, V. Preconditioning CS exposure: Variation in place of conditioning and presentation. Psychonomic Science, 1969, 15, 54-55.

Anderson, D. C., Wolf, D., \& Sullivan, P. Preconditioning exposure to the CS: Variation in place of testing. Psychonomic Science, 1969, 14, 233-235.

Bonkes, R. A. Performance on learning to associate a stimulus with positive reinforcement. In $\mathbf{H}$. Davis \& H. M. B. Hurwitz (Eds.), Operant-Pavlovian interactions. Hillsdale, N.J: Erlbaum, 1977.

Carlton, P. L., \& Vogel, J. R. Habituation and conditioning. Journal of Comparative and Physiological Psychology, 1967, 63, 348-351.

Channell, S., \& Hall, G. Facilitation and retardation of discrimination learning after exposure to the stimuli. Journal of Experimental Psychology: Animal Behavior Processes, 1981, 7, $437-446$.

Dexten, W. R., \& Merrill, H. K. Role of contextual discrimination in fear conditioning. Journal of Comparative and Physiological Psychology, 1969, 69, 677-681.
Domjan, M., \& Stegel, S. Conditioned suppression following CS preexposure. Psychonomic Science, 1971, 25, 11-12.

Halaren, C. R. Latent inhibition in rats: Associative or nonassociative. Journal of Comparative and Physiological Psychology, 1974, 86, 74-78.

Hall, G., \& Minor, H. A search for context-stimulus associations in latent inhibition. Quarterly Journal of Experimental Psychology, in press.

Hall, G., \& Pearce, J. M. Latent inhibition of a CS during CS-US pairings. Journal of Experimental Psychology: Animal Behavior Processes, 1979, 5, 31-42.

Holland, P. C. Conditioned stimulus as a determinant of the form of the Pavlovian conditioned response. Journal of Experimental Psychology: Animal Behavior Processes, 1977, 3, 77-104.

Holland, P. C. Differential effects of omission contingencies on various components of Pavlovian appetitive conditioned responding in rats. Journal of Experimental Psychology: Animal Behavior Processes, 1979, 5, 178-193.

Lubow, R. E. Latent inhibition. Psychological Bulletin, 1973, 79, 398-407.

Lubow, R. E., Markman, R. E., \& Allen, J. Latent inhibition and classical conditioning of the rabbit pinna response. Journal of Comparative and Physiological Psychology, 1969, 66, 688-694.

Lubow, R. E., Rifkin, B., \& Alex, M. The context effect: The relationship between stimulus preexposure and environmental preexposure determines subsequent learning. Journal of Experimental Psychology: Animal Behavior Processes, 1976, 2, 38-47.

Lubow, R. E., Schnur, P., \& Rirkin, B. Latent inhibition and conditioned attention theory. Journal of Experimental Psychology: Animal Behavior Processes, 1976, 2, 163-174.

Mackintosh, N. J. Stimulus selection: Learning to ignore stimuli that predict no change in reinforcement. In $\mathbf{R}$. A. Hinde \& J. S. Hinde (Eds.), Constraints on learning. London: Academic Press, 1973.

Mackintosh, N. J. A theory of attention: Variations in the associability of stimuli with reinforcement. Psychological Review, 1975, 82, 274-298.

Mackintosh, N. J., \& Dickingon, A. Instrumental (type II) conditioning. In A. Dickinson \& R. A. Boakes (Eds.), Mechnisms of learning and motivation. Hillsdale, N.J: Erlbaum, 1979.

Pearce, J. M., \& Hall, G. A model for Pavlovian learning: Variations in the effectiveness of conditioned but not of unconditioned stimuli. Psychological Review, 1980, 87, 532-552.

Reiss, S., \& Whanen, A. R. CS habituation produces a "latent inhibition effect" but no active "conditioned inhibition." Learning and Motivation, 1972, 3, 237-245.

Rescorla, R. A. Summation and retardation tests of latent inhibition. Journal of Comparative and Physiological Psychology, 1971, 75, 77-81.

Rudy, J. W., Krauter, E. E., \& Gafruri, A. Attenuation of the latent inhibition effect by prior exposure to another stimulus. Journal of Experimental Psychology: Animal Behavior Processes, 1976, 2, 235-247.

Sharp, P. E., JAmes, J. H., \& Wagner, A. R. Habituation of a "blocked" stimulus during Pavlovian conditioning. Bulletin of the Psychonomic Society, 1980, 15, 139-142.

Sieqel, S. Generalization of latent inhibition. Journal of Comparative and Physiological Psychology, 1969, 69, 157-159.

SiEgel, S. Latent inhibition and eyelid conditioning. In A. H. Black \& W. F. Prokasy (Eds.), Classical conditioning II: Current research and theory. New York: Appleton-Century-Crofts, 1972.

Tranbero, D. K., \& Rillina, M. Latent inhibition in the autoshaping paradigm. Bulletin of the Psychonomic Society, 1978, 11, 273-276.

Wagner, A. R. Priming in STM: An information processing mechanism for self-generated or retrieval-generated depression in performance. In T. J. Tighe \& R. N. Leaton (Eds.), Habitu- 
ation: Perspectives from child development, animal behavior and neurophysiology. Hillsdale, N.J: Erlbaum, 1976.

WAGNER, A. R. Expectancies and the priming of STM. In S. H. Hulse, H. Fowler, \& W. K. Honig (Eds.), Cognitive processes in animal behavior. Hillsdale, N.J: Erlbaum, 1978.

WAGNER, A. R. Habituation and memory. In A. Dickinson \&
R. A. Boakes (Eds.), Mechanisms of learning and motivation. Hillsdale, N.J: Erlbaum, 1979.

(Manuscript received July 28, 1982;

revision accepted for publication December 2, 1982.)

\section{ANNOUNCEMENT}

Meetings of the Psychonomic Society, 1983-1985

The locations and dates for the next three annual meetings are as follows:

1983

1984

1985
San Diego

San Antonio

Boston
November 17-19

November 1-3

November 22-24 